This paper has been published in Journal of Group Theory, 13(1):143-149 (2010).

Copyright 2010 by Walter de Gruyter.

The final publication is available at www.degruyter.com.

http://dx.doi.org/10.1515/jgt.2009.038

http://www.degruyter.com/view/j/jgth.2010.13.issue-1/jgt.2009.038/

jgt. 2009.038.xml 


\title{
On self-normalising subgroups of finite groups
}

\author{
A. Ballester-Bolinches* $\quad$ R. Esteban-Romero ${ }^{\dagger}$ \\ Yangming $\mathrm{Li}^{\ddagger}$
}

\begin{abstract}
The aim of this paper is to characterise the classes of groups in which every subnormal subgroup is normal, permutable, or S-permutable by the embedding of the subgroups (respectively, subgroups of prime power order) in their normal, permutable, or S-permutable closure, respectively.

Keywords: finite group, permutability, Sylow permutability, permutable closure, subnormal closure, PST-group, PT-group, T-group.

Mathematics Subject Classification (2000): 20D10, 20D20, 20D35

Dedicated to Derek J. S. Robinson on the occasion of his seventieth birthday.
\end{abstract}

\section{Introduction and statement of results}

In this paper, we consider only finite groups.

A subgroup $H$ of a group $G$ is said to be permutable in $G$ if $H$ permutes with every subgroup of $G$. A group $G$ is said to be a PT-group (respectively, $T$-group) if permutability (respectively, normality) is a transitive relation in $G$. By a result of Ore [13], PT-groups are exactly those groups where all subnormal subgroups are permutable. PST-groups are also defined via a transitivity property, namely with respect to S-permutability ([11]): a subgroup of a group $G$ is called $S$-permutable if it permutes with all the Sylow

${ }^{*}$ Departament d'Àlgebra, Universitat de València, Dr. Moliner, 50, E-46100 Burjassot, València, Spain, email: Adolfo.Ballester@uv.es

${ }^{\dagger}$ Departament de Matemàtica Aplicada-IUMPA, Universitat Politècnica de València, Camí de Vera, s/n, E-46022 València, Spain, email: resteban@mat.upv.es

${ }^{\ddagger}$ Department of Mathematics, Guangdong College of Education, Guangzhou, 510310, People's Republic of China, email: liyangming@gdei.edu.cn 
subgroups of $G$. By a result of Kegel ([11, Satz 1]), every S-permutable subgroup is subnormal and hence $P S T$-groups are exactly those groups in which all subnormal subgroups are S-permutable.

Note that the class of $T$-groups is a proper subclass of the class of $P T$ groups, which in turns forms a proper subclass of the class of PST-groups. These classes have been studied in detail, with many characterisations available (see $[1,2,3,4,5,6,7,14,15]$ ).

The basic structure of soluble T-, PT-, and PST-groups were established by Gaschütz, Zacher, and Agrawal, respectively, and are presented in the following theorem.

Theorem 1. Let $L$ be the nilpotent residual of a group $G$. Then:

1. (Agrawal, [1]) $G$ is a soluble PST-group if and only if $L$ is an abelian Hall subgroup of odd order of $G$ on which $G$ acts by conjugation as a group of power automorphisms.

2. (Zacher, [15]) $G$ is a soluble PT-group if and only if $G$ is a soluble PST-group with modular Sylow subgroups.

3. (Gaschütz, [10]) $G$ is a soluble T-group if and only if $G$ is a soluble PST-group with Dedekind Sylow subgroups.

The results of the present paper spring from a recent characterisation of soluble $T$-groups due to the third author. There he proves that a group is a soluble $T$-group if and only if every subgroup is self-normalising in its normal closure ([12, Theorem 3.1]). It is natural to wonder whether or not it is possible to get similar characterisations of soluble PT-and PST-groups by using permutable and S-permutable versions of the normal closure. This is the goal of the present paper.

Since the intersection of S-permutable subgroups of a group $G$ is again a Spermutable subgroup of $G$, it seems reasonable to replace the normal closure of a subgroup $H$ by the intersection of all S-permutable subgroups of $G$ containing $H$ in the PST-case. Unfortunately, the intersection of permutable subgroups of $G$ is not permutable in general. Therefore the intersection of all permutable subgroups of $G$ containing a given subgroup $H$ is not the smallest permutable subgroup of $G$ containing $H$ in general. This is the main reason why the proofs concerning $P T$-groups are much more involved than the corresponding ones for $T$ - and $P S T$-groups. Despite this fact, the above subgroup will play a central role in our approach.

Definition 2. Let $H$ be a subgroup of a group $G$. 
1. The permutable closure $\mathrm{A}_{G}(H)$ of $H$ in $G$ is the intersection of all permutable subgroups of $G$ containing $H$.

2. The S-permutable closure $\mathrm{B}_{G}(H)$ of $H$ in $G$ is the intersection of all S-permutable subgroups of $G$ containing $H$.

Applying [11], $\mathrm{B}_{G}(H)$ is S-permutable in $G$. However, $\mathrm{A}_{G}(H)$ is not permutable in general, as the following example shows:

Example 3. Suppose that $p$ is a prime and $m>1$ is a natural number. Let $G=\left\langle a, b, c \mid a^{p^{m}}=b^{p}=c^{p}=1, b^{a}=b c, c^{a}=c^{b}=c\right\rangle$. The subgroups $H_{1}=\langle b, c\rangle$ and $H_{2}=\left\langle b, c a^{p^{m-1}}\right\rangle$ are permutable in $G$, but the intersection $H=H_{1} \cap H_{2}$ is not permutable in $G$. For the subgroup $H$, the permutable closure $\mathrm{A}_{G}(H)=H$ is not permutable in $G$.

However we have:

Theorem 4. Assume that $G$ is a group such that every subgroup is selfnormalising in its permutable closure. Then $\mathrm{A}_{G}(H)$ is a permutable subgroup of $G$ for each subgroup $H$ of $G$.

Theorem 4 is a consequence of a stronger result:

Theorem 5. Let $p$ be a prime. If every p-subgroup of a group $G$ is selfnormalising in its permutable closure, then $\mathrm{A}_{G}(H)$ is a permutable subgroup of $G$ for every p-subgroup $H$ of $G$.

These results justify the study of the class of groups in which every subgroup is self-normalising in its permutable closure. It turns out that this class is the class of all soluble PT-groups.

If we fix a prime $p$, the class of groups for which every $p$-subgroup is self-normalising in its permutable closure is a subclass of a class which can be considered as a local version of the class of all soluble PT-groups.

Definition 6 ([5]). A group $G$ satisfies $\mathcal{X}_{p}$ if and only if each subgroup of a Sylow $p$-subgroup $P$ of $G$ is permutable in the normaliser $\mathrm{N}_{G}(P)$.

Theorem 7. If $p$ is a prime and $G$ is a group in which every p-subgroup is self-normalising in its permutable closure, then $G$ satisfies $\mathcal{X}_{p}$.

Theorem 8. For a group $G$, the following statements are equivalent:

1. $G$ is a soluble PT-group.

2. $H$ is abnormal in $\mathrm{A}_{G}(H)$ for every subgroup $H$ of $G$. 
3. $\mathrm{N}_{G}(H) \cap \mathrm{A}_{G}(H)=H$ for every subgroup $H$ of $G$.

4. For every prime $p$ and every p-subgroup $H$ of $G, H$ is abnormal in $\mathrm{A}_{G}(H)$.

5. For every prime $p$ and every $p$-subgroup $H$ of $G$, we have $\mathrm{N}_{G}(H) \cap$ $\mathrm{A}_{G}(H)=H$.

Theorem 7 follows from the local strategy we use in the PST-case. In fact, this local point of view leads to the local defining property of the class of soluble PST-groups.

Recall that if $p$ is a prime, a group $G$ satisfies property $\mathcal{Y}_{p}$ if for each pair of $p$-subgroups $H$ and $K$ of $G$ such that $H$ is contained in $K$, then $H$ is S-permutable in $\mathrm{N}_{G}(K)([4])$. A group $G$ satisfies $\mathcal{C}_{p}$ if every subgroup of a Sylow $p$-subgroup $P$ of $G$ is normal in $\mathrm{N}_{G}(P)$ ([14]).

A group $G$ is a soluble $P S T$-group if and only if $G$ satisfies $\mathcal{Y}_{p}$ for all primes ([4, Theorem 4]). Similar results hold for soluble $P T$-groups and property $\mathcal{X}_{p}([5])$, and soluble $T$-groups and property $\mathcal{C}_{p}([14])$. These results are consequences of the following:

Theorem 9 ([4, Theorem 3]). Let $p$ be a prime. A group $G$ satisfies $\mathcal{X}_{p}$ (respectively, $\mathcal{C}_{p}$ ) if and only if $G$ satisfies $\mathcal{Y}_{p}$ and the Sylow p-subgroups of $G$ are modular (respectively, Dedekind).

If $p$ is a prime and every $p$-subgroup is self-normalising in its permutable closure, then Sylow subgroups are modular. Hence Theorem 7 , by virtue of Theorem 9, is the permutable local version of the following:

Theorem 10. If $p$ is a prime and $G$ is a group such that every p-subgroup of $G$ is self-normalising in its $S$-permutable closure, then $G$ satisfies $\mathcal{Y}_{p}$.

The converse of the above result does not hold.

Example 11. Suppose that $p$ and $q$ are two primes such that $q$ divides $p-1$. Let $E$ be an extraspecial group of order $p^{3}$ and exponent $p$. Let $i$ and $j$ be two numbers such that $i j \equiv 1(\bmod p-1)$ and $i$ and $j$ have order $q$ modulo $p$. Let $\{x, y\}$ be a generating system for $E$ and let $z$ be an automorphism of order $q$ of $E$ given by $x^{z}=x^{i}, y^{z}=y^{j}$. Let $G=[E]\langle z\rangle$ be the corresponding semidirect product, then the S-permutable closure of $Z=\langle z\rangle$ is $G$, but $\mathrm{N}_{G}(Z)=\langle z,[x, y]\rangle$. However, the group $G$ satisfies $\mathcal{Y}_{q}$ because it is $q$-nilpotent.

Note that if $H$ is a $p$-subgroup of $G$ and $\mathrm{N}_{G}(H) \cap\left\langle H^{G}\right\rangle=H$, where $\left\langle H^{G}\right\rangle$ is the normal closure of $H$ in $G$, we have that $H$ is a Sylow $p$-subgroup 
of $\left\langle H^{G}\right\rangle$. Hence if the above condition holds for every $p$-subgroup of $G$, it follows that the Sylow $p$-subgroups of $G$ are Dedekind groups. Therefore applying Theorems 9 and 10 we have:

Corollary 12. Let $p$ be a prime and let $G$ be a group. If every p-subgroup of $G$ is self-normalising in its normal closure, then $G$ satisfies property $\mathcal{C}_{p}$.

We are now in a position to give characterisations of groups in which every subgroup is self-normalising in its S-permutable closure: they turn out to be the soluble PST-groups.

Theorem 13. Let $G$ be a group. Any two of the following five statements are equivalent:

1. $G$ is a soluble PST-group.

2. For every subgroup $H$ of $G, H$ is abnormal in $\mathrm{B}_{G}(H)$.

3. For every subgroup $H$ of $G$, the equality $\mathrm{N}_{G}(H) \cap \mathrm{B}_{G}(H)=H$ holds.

4. If $p$ is any prime and $H$ is a p-subgroup of $G$, then $H$ is abnormal in $\mathrm{B}_{G}(H)$.

5. If $p$ is any prime and $H$ is a p-subgroup of $G$, it follows that $\mathrm{N}_{G}(H) \cap$ $\mathrm{B}_{G}(H)=H$.

Combining Theorem 13 and Corollary 12, we have:

Corollary 14 ([12, Theorem 3.1]). Any two of the following assertions about a group $G$ are equivalent:

1. $G$ is a soluble T-group.

2. $H$ is abnormal in $\left\langle H^{G}\right\rangle$ for all subgroups $H$ of $G$.

3. $\mathrm{N}_{G}(H) \cap\left\langle H^{G}\right\rangle=H$ for all subgroups $H$ of $G$.

4. $H$ is abnormal in $\left\langle H^{G}\right\rangle$ for every $p$-subgroup $H$ of $G$ and every prime $p$.

5. $\mathrm{N}_{G}(H) \cap\left\langle H^{G}\right\rangle=H$ for every $p$-subgroup $H$ of $G$ and every prime $p$. 


\section{Proofs}

We begin the section with a pair of lemmas, which will be used several times in subsequent proofs.

Lemma 15. Let $G$ be a group and let $N$ be a normal subgroup of $G$. If $H$ is a subgroup of $G$, then $\mathrm{A}_{G}(H) N / N$ and $\mathrm{B}_{G}(H) N / N$ are subgroups of $\mathrm{A}_{G / N}(H N / N)$ and $\mathrm{B}_{G / N}(H N / N)$, respectively. If $N$ is contained in $\mathrm{A}_{G}(H)$, then $\mathrm{A}_{G}(H) / N=\mathrm{A}_{G / N}(H N / N)$. The same is true for $\mathrm{B}_{G}(H)$.

Proof. The assertions made in the lemma follow from the fact that a subgroup $X$ of $G$ containing $N$ is permutable (respectively, S-permutable) in $G$ if and only if $X / N$ is permutable (respectively, S-permutable) in $G / N$.

Lemma 16. Let $H$ be a subgroup of a group $G$ and let $S$ be a subgroup of $G$ containing $H$. Then $\mathrm{A}_{S}(H) \leq \mathrm{A}_{G}(H)$ and $\mathrm{B}_{S}(H) \leq \mathrm{B}_{G}(H)$.

Proof. Note that if $X$ is a permutable (respectively, S-permutable) subgroup of $G$ containing $H$, then $X \cap S$ is a permutable (respectively, S-permutable) subgroup of $S$ containing $H$.

Corollary 17. The classes of all groups in which every subgroup is selfnormalising in its permutable (respectively, S-permutable) closure are closed under taking subgroups and factor groups.

Proof of Theorem 5. Assume that every $p$-subgroup of $G$ is self-normalising in its permutable closure. We prove that $\mathrm{A}_{G}(H)$ is permutable in $G$ by induction on the order of $G$. If $N$ is a minimal normal subgroup of $G$ contained in $\mathrm{A}_{G}(H)$, then $\mathrm{A}_{G}(H) / N=\mathrm{A}_{G / N}(H N / N)$ by Lemma 15 . Since the hypotheses of the theorem hold in $G / N$, we have that $\mathrm{A}_{G}(H) / N$ is permutable in $G / N$ by induction. Hence $\mathrm{A}_{G}(H)$ is permutable in $G$, as required. Therefore we can suppose that $\operatorname{Core}_{G}\left(\mathrm{~A}_{G}(H)\right)=1$. On the other hand, applying [11, Satz 2], $\mathrm{A}_{G}(H)$ is S-permutable in $G$ and so $\mathrm{A}_{G}(H)$ is nilpotent by a result of Deskins [8, Theorem 1]. Since $H$ is self-normalising in $\mathrm{A}_{G}(H)$, it follows that $\mathrm{A}_{G}(H)$ is a $p$-group and so $H=\mathrm{A}_{G}(H)$ is S-permutable in $G$. By [11, Satz 1], $H$ is subnormal in $G$. Let $P$ be a Sylow $p$-subgroup of $G$. Then $H$ is contained in $P$. If $P$ were a proper subgroup of $G$, then $H$ would be permutable in $P$ because $P$ inherits the hypotheses of the theorem. It would imply the permutability of $H$ in $G$, as required. Hence we may assume that $P=G$. Then a minimal normal subgroup $N$ of $G$ is central. Since $H$ is self-normalising in $G$, it follows that $N$ must be contained in $H$, contradicting the fact that $\operatorname{Core}_{G}(H)=1$. Therefore $H$ is permutable in $P$ and so in G. 
Proof of Theorem 10. Let $H$ and $K$ be $p$-subgroups of $G$ such that $H$ is contained in $K$ and let $P$ be a Sylow $p$-subgroup of $G$ containing $K$. Write $T=\mathrm{N}_{G}(K)$. We must prove that $H$ is S-permutable in $T$. Applying Lemma $16, H$ is self-normalising in $\mathrm{B}_{T}(K)$. If $H$ is not S-permutable in $T$, then $H$ is a proper subgroup of $\mathrm{B}_{T}(H)$. It implies that $H$ is a proper subgroup of its normaliser in $\mathrm{B}_{T}(H)$ as $H$ is a subnormal subgroup of $T$. This contradiction shows that $H$ is S-permutable in $\mathrm{N}_{G}(K)$ and $G$ has property $\mathcal{Y}_{p}$.

Proof of Theorem 13. 1 implies 2. We suppose that the implication is not true and derive a contradiction. Let $(G, H)$ be a counterexample with $|G|$ minimal. Assume that $A=\mathrm{B}_{G}(H)$ is a proper subgroup of $G$. Then, by Theorem $1, A$ is a soluble $P S T$-group and so $H$ is abnormal in $\mathrm{B}_{A}(H)$. Since $\mathrm{B}_{A}(H)$ is S-permutable in $A$ and $A$ is S-permutable in $G$, it follows that $\mathrm{B}_{A}(H)$ is S-permutable in $G$ because $G$ is a $P S T$-group. Consequently $A=\mathrm{B}_{A}(H)$ and then $H$ is abnormal in $A$. This contradiction shows that $A=G$. If $N:=\operatorname{Core}_{G}(H) \neq 1$, we have that $H / N$ is abnormal in $G / N$ by the minimal choice of $G$. Hence $H$ is abnormal in $G$. Thus we can assume that $N:=\operatorname{Core}_{G}(H)=1$. Applying Agrawal's theorem (Theorem 1), $G=L M$, where $L$ is the nilpotent residual of $G, L \cap M=1$, and $L$ is an abelian normal Hall subgroup of $G$ of odd order acted upon by conjugation as a group of power automorphisms by $M$. It implies that every subgroup of $L$ is normal in $G$ and so $L \cap H=1$. Hence we can assume, without loss of generality, that $H$ is contained in $M$. Since $M$ is nilpotent, we have that $L H$ is S-permutable in $G$. Thus $\mathrm{B}_{G}(H)=G=L H$ and $H=M$. Applying [9, IV, 5.18 and III, 4.6], $H$ is a Carter subgroup of $G$. Applying [9, IV, Section 3 and 4.6, and I, 6.21], $H$ is abnormal in $G$. This is the desired contradiction.

On the other hand, by virtue of [9, I, 6.21], every abnormal subgroup is self-normalising. Therefore 2 implies 3 and 4 implies 5 . It is clear that 2 implies 4 and 3 implies 5 .

To complete the proof we now show that 5 implies 1 . Assume that every $p$-subgroup is self-normalising in its S-permutable closure for each prime $p$. By Theorem 10, $G$ satisfies $\mathcal{Y}_{p}$ for each prime $p$. Applying [4, Theorem 4], we conclude that $G$ is a soluble $P S T$-group.

Proof of Theorem 8. The same arguments to those used in the proof of Theorem 13 replacing Agrawal's result by Zacher's result (see Theorem 1) show that 1 implies 2. It is clear that 2 implies 3 and 4 implies 5 . Obviously 2 implies 4 and 3 implies 5 .

Now if every $p$-subgroup is self-normalising in its permutable closure for each prime $p$, then $G$ satisfies $\mathcal{X}_{p}$ for each prime $p$ by virtue of Theorem 7 . Applying [5, Theorem A], $G$ is a soluble PT-group. 


\section{Acknowledgements}

This research has been supported by the grants MTM2004-08219-C02-02 and MTM2007-68010-C03-02 from MEC (Spanish Government) and FEDER (European Union) and GV/2007/243 from Generalitat (Valencian Community).

\section{References}

[1] R. K. Agrawal. Finite groups whose subnormal subgroups permute with all Sylow subgroups. Proc. Amer. Math. Soc., 47(1):77-83, 1975.

[2] M. J. Alejandre, A. Ballester-Bolinches, and M. C. Pedraza-Aguilera. Finite soluble groups with permutable subnormal subgroups. J. Algebra, 240(2):705-722, 2001.

[3] A. Ballester-Bolinches and R. Esteban-Romero. Sylow permutable subnormal subgroups of finite groups II. Bull. Austral. Math. Soc., 64(3):479-486, 2001.

[4] A. Ballester-Bolinches and R. Esteban-Romero. Sylow permutable subnormal subgroups of finite groups. J. Algebra, 251(2):727-738, 2002.

[5] J. C. Beidleman, B. Brewster, and D. J. S. Robinson. Criteria for permutability to be transitive in finite groups. J. Algebra, 222(2):400-412, 1999 .

[6] J. C. Beidleman, H. Heineken, and M. F. Ragland. Strong Sylow bases and mutually permutable products. Preprint, 2008.

[7] R. A. Bryce and J. Cossey. The Wielandt subgroup of a finite soluble group. J. London Math. Soc., 40(2):244-256, 1989.

[8] W. E. Deskins. On quasinormal subgroups of finite groups. Math. Z., 82:125-132, 1963.

[9] K. Doerk and T. Hawkes. Finite Soluble Groups, volume 4 of De Gruyter Expositions in Mathematics. Walter de Gruyter, Berlin, New York, 1992.

[10] W. Gaschütz. Gruppen, in denen das Normalteilersein transitiv ist. J. reine angew. Math., 198:87-92, 1957.

[11] O. H. Kegel. Sylow-Gruppen und Subnormalteiler endlicher Gruppen. Math. Z., 78:205-221, 1962. 
[12] Y. Li. Finite groups with NE-subgroups. J. Group Theory, 9:49-58, 2006. 2006.

[13] O. Ore. Contributions to the theory of groups of finite order. Duke Math. J., 5:431-460, 1939.

[14] D. J. S. Robinson. A note on finite groups in which normality is transitive. Proc. Amer. Math. Soc., 19:933-937, 1968.

[15] G. Zacher. I gruppi risolubli finiti in cui i sottogruppi di composizione coincidono con i sottogrupi quasi-normali. Atti Accad. Naz. Lincei Rend. cl. Sci. Fis. Mat. Natur. (8), 37:150-154, 1964. 